Bull Yamaguchi Med School 53 (3-4):19-23, 2006

\title{
Ryanodine Receptor Defects in Heart Failure
}

\author{
Tetsuro Oda, Masafumi Yano and Masunori Matsuzaki
}

Department of Medicine and Clinical Science, Division of Cardiology, Yamaguchi University Graduate School of Medicine, 1-1-1, Minami-Kogushi, Ube, Yamaguchi 755-8505, Japan

(Received September 26, 2006)

\begin{abstract}
Abnormal regulation of intracellular $\mathrm{Ca}^{2+}$ by sarcoplasmic reticulum plays a part in the mechanism underlying contractile and relaxation dysfunction in heart failure $(\mathrm{HF})$. The protein-kinase-A-mediated hyperphosphorylation of ryanodine receptors in the sarcoplasmic reticulum has been shown to cause the dissociation of FKBP12.6 (also known as calstabin-2) from ryanodine receptors in HF. In addition, several disease-linked mutations in the ryanodine receptors have been reported in patients with catecholaminergic polymorphic ventricular tachycardia or arrhythmogenic right ventricular cardiomyopathy type2. The unique distribution of these mutation sites has led to the concept that the interaction among the putative regulatory domains within the ryanodine receptors has a key role in regulating channel opening. The knowledge gained from various studies of ryanodine receptors under pathologic conditions might lead to the development of new pharmacological or genetic strategies for the treatment of HF or cardiac arrhythmia. In this review, we focus on the role of the $\mathrm{Ca}^{2+}$-release channel, the ryanodine receptor, in the pathogenesis of HF and fatal arrhythmia, and the possibility of developing new therapeutic strategies by targeting this receptor.
\end{abstract}

Key words: heart failure, ryanodine receptor, excitation-contraction coupling, domain Interaction

\section{Introduction}

Regardless of the initial cause of myocardial damage - for example, hypertension, myocardial ischemia or cardiomyopathy heart failure (HF) eventually occurs in parallel with the activation of neurohumoral factors if the damage persists for a long period of time. ${ }^{1)}$ A substantial amount of evidence suggests that the abnormal regulation of intracellular $\mathrm{Ca}^{2+}$ by the sarcoplasmic reticulum plays a key part in the development of $\mathrm{HF}^{2)}$ In many cases, altered $\mathrm{Ca}^{2+}$ cycling causatively affects the depression of mechanical performance and, consequently, improvement of $\mathrm{Ca}^{2+}$ cycling has potential as a new therapeutic strategy against $\mathrm{HF}^{3)}$ In this review, we focus on the role of the $\mathrm{Ca}^{2+}$-release channel, referred to as the ryanodine receptor (RyR), in the pathogenesis of HF and fatal arrhythmia, and on the possibility of developing a new therapeutic strategy by targeting this receptor.

\section{Concept of intramolecular domain unzipping}

Missense mutations in the homologous RyR1 channel gene that have been linked to diseases of the skeletal muscle, including malignant hyperthermia $(\mathrm{MH})$ and central core disease (CCD), cluster into three well-known regions of the RyR 1 channel that correspond to the mutable regions of the RyR2 channel seen in patients with (Arrhythmogenic right ventricular cardiomyopathy (ARVC) or (Catecholaminergic polymorphic ventricular 
tachycardia (CPVT).

Under experimental conditions, $\mathrm{MH} \mathrm{mu}-$ tant RyR1 channels display a phenotype similar to CPVT mutations in RyR2, hyperactivity under resting conditions, and $\mathrm{Ca}^{2+}$ leak from the SR into the cytoplasm. Ikemoto and colleagues have proposed that N-terminal and central domains in RyR1 contribute to the conformational stabilization of the closed state of the channel via zipped interaction of these two domains. ${ }^{4) 5}$

More simply put, they predict that sequences of the channel that are not adjacent to one another in the linear peptide sequence can interact once the channel is folded into its 3-dimensional structure. Furthermore, Ikemoto and colleagues have proposed that channel opening during excitation-contraction coupling requires domain unzipping to reduce the conformational energy constraints necessary to induce the open state. ${ }^{5) 6}$ According to the domain-zipping model, $\mathrm{MH}$-associated mutations occurring in either the N-terminal or central domains of RyR 1 reduce the affinity of the zipper domains, resulting in leaky channels.

\section{Defective intermolecular domain interactions in the cardiac RyR2 isoform and correlation with structural studies}

Because skeletal RyR1 and cardiac RyR2 are $66 \%$ homologous at the protein level, it was proposed that domain unzipping may also occur in the cardiac $\mathrm{Ca}^{2+}$-release channel RyR2. We have designed a synthetic peptide (DPc10), corresponding to Gly2460-Pro2495 of RyR2. Binding of the peptide DPc10 to the hypothetical $\mathrm{N}$-terminal zipper domain of $\mathrm{Ry}$ $\mathrm{R} 2$ was found to increase sensitivity to $\mathrm{Ca}^{2+}$ -dependent channel activation and diastolic SR $\mathrm{Ca}^{2+}$ leak to levels observed in failing hearts. In contrast, introduction of the CPVT-associated RyR2 mutation Arg2474Ser into DPc10 (DPc10-mut) abolished these effects indirectly, suggesting that the DPc10mut peptide, which was not able to interfere with the interdomain interaction or to produce domain unzipping effects, may mimic effects of the identical CPVT mutation in the RyR2 channel. To further test the hypothesis that DPc10 produces unzipping of interacting
N-terminal and central domains in RyR2, RyR2 was fluorescently labeled with MCA using DPc10 as a site-directing carrier.

Site-specific antibody labeling of calpaindigested RyR2 fragments revealed that MCA fluorescence bound to the $\mathrm{N}$-terminal $120 \mathrm{kDa}$ of RyR2. These data support the idea that the N-terminal and central domains of RyR2 interact with each other. Interestingly, the clamp structure containing the $\mathrm{N}$-terminus projection was shown to undergo conformational changes corresponding to channel opening and closing ${ }^{7) 8}$ and appears to be localized near the FKBP12 binding region. ${ }^{9)}$ Because others group have shown that FKBP12 binds to the central domain of the full-length RyR1 involving the critical amino acid Val2461 corresponding to Ile2427 in the cardiac RyR2, ${ }^{1011)}$ the structural and experimental data provide further support for the concept of $\mathrm{N}$-terminal and central domain interaction. Because of the high degree of sequence identity, it is likely that RyR1 intradomain mechanisms and interactions with FKBP12 (calstabin1) similarly apply to the cardiac RyR2 and FKBP12.6. Further support for the hypothesis of inter-domain interaction between N-terminal and central domains within RyR2 was obtained by chemical quenching assay using the bulky fluorescence quencher QSY-BSA. The rationale of peptide targeting of the MCA fluorescent probe to the N-terminus of RyR2 was used in combination with the large fluorescence quencher to monitor accessibility of unzipped domain configurations. Quenching extent of MCA fluorescence by large chemical quencher QSY-BSA was considerably increased after treatment with DPc10 or cAMP (which increases PKA phosphorylation of RyR2 at Ser2809). ${ }^{12)}$ Accessibility of the QSY-BSA quencher was also significantly increased in SR preparations from failing hearts, which indicates domain unzipping of the regulatory domains resulting from PKA hyperphosphorylation of RyR2. ${ }^{13)}$ Under physiological conditions, stress or exercise result in transiently increased PKA phosphorylation of RyR2 at Ser2809, which transiently decreases the binding affinity of FKBP12.6 (calstabin2) to RyR2 and increases $\mathrm{Ca}^{2+}$ dependent activation of the channel. ${ }^{12(14) 15)}$ 
Thus, after PKA phosphorylation of RyR2, there is increased $\mathrm{SR} \mathrm{Ca}^{2+}$ release for any given trigger $\left[\mathrm{Ca}^{2+}\right] \mathrm{i}{ }^{14) 15)}$ On the other hand, chronic PKA hyperphosphorylation of RyR2 in $\mathrm{HF}$ results in incomplete channel closure during diastole, which causes depletion of the SR $\mathrm{Ca}^{2+}$ store and reduced systolic $\mathrm{Ca}^{2+}$ release. ${ }^{13) 1417)}$ Experiments with the QSY-BSA fluorescence quencher revealed that DPc10induced domain unzipping does not induce calstabin2 dissociation by itself. Rather, These data suggest that domain unzipping may facilitate PKA phosphorylation of RyR2 and hence may enhance calstabin2 dissociation in response to PKA phosphorylation of RyR2. Peptide-induced domain unzipping would increase the accessibility of the PKA catalytic subunit (which is bound to RyR2 via the targeting protein $\mathrm{mAKAP}$ ) to the PKA phosphorylation site Ser2809 on RyR2. ${ }^{18)} \mathrm{Al}-$ ternatively, under physiological conditions PKA phosphorylation may be expected to induce conformational changes in RyR2 consistent with domain unzipping. Determination of the exact role of domain unzipping in the gating mechanism and regulation of RyR2 will require further studies. Such studies may also reveal how conformational changes in RyR2 resulting from PKA phosphorylation and domain unzipping reduce FKBP12.6 binding affinity to the channel complex.

\section{Leaky RyR2 channels as a therapeutic target for heart failure}

This study provides additional confirmation that abnormal RyR2 channel function in failing hearts is caused by PKAhyperphosphorylation of the channel, followed by dissociation of FKBP12.6 from RyR2. More importantly, domain unzipping was found to be a source mechanism of RyR2 channel dysfunction in HF. In our study, JTV519 inhibited both the FK506-induced $\mathrm{Ca}^{2+}$ leak from the RyR2 in normal sarcoplasmic reticulum and the spontaneous $\mathrm{Ca}^{2+}$ leak in failing sarcoplasmic reticulum isolated from a pacing induced dog HF model. No abnormal C $\mathrm{a}^{2+}$ leak was noted in the sarcoplasmic reticular vesicles isolated from JTV519-treated hearts; the amount of the RyR2-bound
FKBP12.6 was restored to a normal level. In JTV519-untreated hearts, the RyR2 was PKA-hyperphosphorylated, whereas the opposite was seen in JTV519-treated hearts, in which the channel phosphorylation was returned to levels similar to those seen in normal hearts. We reported that JTV519 restored the zipped state of interactions between domains within RyR2, which prevented $\mathrm{Ca}^{2+}$ leak from occurring in failing sarcoplasmic reticulum isolated from a pacing-induced dog HF model. ${ }^{19)}$ These beneficial effects of JTV519 were not mediated by rebinding of (RyR-unbound) FKBP12.6. ${ }^{19)}$ This finding suggests that the primary mechanism of pharmacological action of JTV519 is to stabilize the interactions between domains and, in turn, prevent FKBP12.6 dissociation and $\mathrm{Ca}^{2+}$ leak (Fig. 1). Further study is clearly needed to find out whether JTV519 is still effective for modulation of RyR2 channel gating in FKBP12.6-depleted (by PKAphosphorylation or FK506) RyR2. Overall, although uncertainty still exists about the mechanism by which JTV519 stabilizes RyR2 channel gating in failing hearts, the above data suggest that stabilization of the RyR2 represents a new molecular target for the treatment or prevention of exercise-induced arrhythmias and sudden death in patients with catecholaminergic polymorphic ventricular tachycardia mutations and $\mathrm{HF}$.

\section{References}

1) Braunwald, E. and Bristow, M.R.: Congestiveheart failure; fifty years of progress. Circulation, 102 : IV14-IV23, 2000.

2) Hasenfuss, G. and Pieske, B.: Calcium cycling in congestive heart failure. J. Mol. Cell Cardiol., 34 : 951-969, 2002.

3 ) Yano, M., et al.: Altered intracellular $\mathrm{Ca}^{2+}$ handlingin heart failure. J. Clin. Invest., $115:$ 556-564, 2005.

4) Yamamoto, T., El-Hayek, R. and Ikemoto, N.: Postulated role of interdomain interaction within the ryanodine receptor in $\mathrm{Ca}^{2+}$ channel regulation. J. Biol. Chem., $275:$ 11618-11625, 2000.

5 ) Ikemoto, N. and Yamamoto, T.: Postulated role of inter-domain interaction within the ryanodine receptor in $\mathrm{Ca}^{2+}$ 


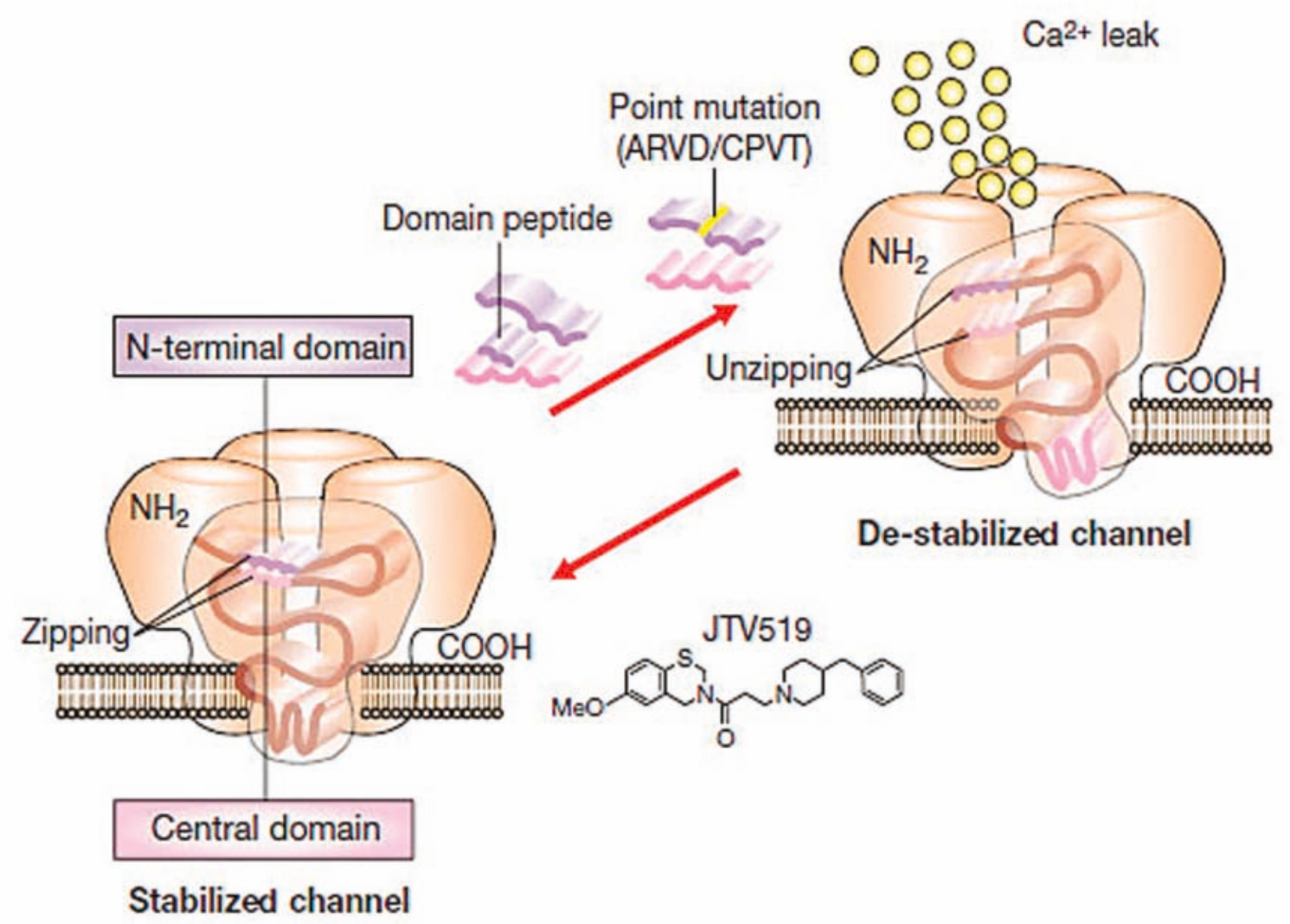

Fig. 1 Schematic diagram of domain-domain interaction within the ryanodine receptor as a new therapeutic target. The N-terminal and central domain interact with each other to function as a regulatory switch for channel gating, with a tight zipping of the interacting domains serving to stabilize the channel. A mutation in either domain weakens the interdomain interaction (unzipping), which causes activation and leakiness of the calcium ion channel. Synthetic domain peptides corresponding to key subdomains of the ryanodine receptor 2 are capable of mimicking diseased conditions of the ryanodine receptor 2 channel by interfering with the interactions between domains. The new 1,4-benzothiazepin derivative, JTV519, directly restores the zipped state of the interdomain interaction within the ryanodine receptor 2, in turn stabilizing the channel gating. ARVD, arrhythmogenic right ventricular cardiomyopathy type 2; CPVT, catecholaminergic polymorphic ventricular tachycardia.

channel regulation. Trends Cardiovasc. Med., $10: 310-316,2000$.

6 ) Ikemoto, N. and Yamamoto, T.: Regulation of calcium release by interdomain interaction within ryanodine receptors. Front. Biosci., 7 : d671- d683, 2002.

7 ) Orlova, E.V., Serysheva, II., van Heel, M., Hamilton, S.L. and Chiu, W.: Two structural configurations of the skeletal muscle calcium release channel. Nat. Struct. Biol., 3 : 547-552, 1996.

8 ) Serysheva, II., Schatz, M., van Heel, M.
Chiu, W. and Hamilton, S.L.: Structure of the skeletal muscle calcium release channel activated with $\mathrm{Ca}^{2+}$ and AMPPCP. Biophys. J., 77 : 1936-1944, 1999.

9) Wagenknecht, T., Radermacher, M., Grassucci, R., Berkowitz, J., Xin, H.B. and Fleischer, S.: Locations of calmodulin and FK506-binding protein on thethreedimensional architecture of the skeletal muscle ryanodine receptor. J. Biol. Chem., 272 : 32463-32471, 1997.

10) Gaburjakova, M., Gaburjakova, J., Rei- 
ken, S., Huang, F., Marx, S.O., Rosemblit, N. and Marks, A.R.: FKBP12 binding modulates ryanodine receptor channel gating. J. Biol. Chem., 276 : 16931 -16935, 2001.

11) Bultynck, G., Rossi, D., Callewaert, G., Missiaen, L., Sorrentino, V., Parys, J.B. and De Smedt, H.: The conserved sites for the FK506-binding proteins in ryanodine receptors and inositol 1,4,5-trisphosphate receptors are structurally and functionally different. J. Biol. Chem., 276 : 4771547724, 2001.

12) Wehrens, X.H., Lehnart, S.E., Reiken, S.R. and Marks, A.R.: $\mathrm{Ca}^{2+}$ calmodulindependent protein kinase II phosphorylation regulates the cardiac ryanodine receptor. Circ. Res., 94 : e61-e70, 2004.

13) Marx, S.O., Reiken, S., Hisamatsu, Y., Jayaraman, T., Burkhoff, D., Rosemblit, N. and Marks, A.R.: PKA phosphorylation dissociates FKBP12.6 from the calcium release channel (ryanodine receptor); defective regulation in failing hearts. Cell, 101 : 365-376, 2000.

14) Wehrens, X.H., Lehnart, S.E., Huang, F., Vest, J.A., Reiken, S.R., Mohler, P.J., Sun, J., Guatimosim, S., Song, L.S., Rosemblit, N., D'Armiento, J.M., Napolitano, C., Memmi, M., Priori, S.G., Lederer, W.J. and Marks, A.R.: FKBP12.6 deficiency and defective calcium release channel (ryanodine receptor) function linked to exercise-induced sudden cardiac death. Cell, $113:$ 829-840, 2003.

15) Wehrens, X.H., Lehnart, S.E., Reiken, S.R., Deng, S.X., Vest, J.A., Cervantes, D., Coromilas, J., Landry, D.W. and Marks, A.R.: Protection from cardiac arrhythmia through ryanodine receptorstabilizing protein calstabin2. Science, 304 : 292-296, 2004.

16) Shannon, T.R., Pogwizd, S.M. and Bers, D. M.: Elevated sarcoplasmic reticulum $\mathrm{Ca}^{2+}$ leak in intact ventricular myocytes from rabbits in heart failure. Circ. Res., 93 : 592-594, 2003.

17) Pieske, B., Maier, L.S., Bers, D.M. and Hasenfuss, G.: $\mathrm{Ca}^{2+}$ handling and sarcoplasmic reticulum $\mathrm{Ca}^{2+}$ content in isolated failing and nonfailing human myocardium. Circ. Res., 85 : 38-46, 1999.
18) Marx, S.O., Reiken, S., Hisamatsu, Y., Gaburjakova, M., Gaburjakova, J., Yang, Y.M., Rosemblit, N. and Marks, A.R.: Phosphorylation-dependent regulation of ryanodine receptors; a novel role for leucine/isoleucine zippers. J. Cell. Biol., $153:$ 699-708, 2001.

19) Oda, T., et al.: Defective regulation of interdomain interactions within the ryanodine receptor plays a key role in the pathogenesis of heart failure. Circulation, 111 : 3400-3410, 2005. 